\title{
Relapse of Rectal Cancer in an Anal Fistula: A Rare Case
}

\author{
RIKAKO KATO, YUJI MIYAMOTO, HIROSHI SAWAYAMA, \\ KATSUHIRO OGAWA, MASAAKI IWATSUKI, SHIRO IWAGAMI, YOSHIFUMI BABA, \\ NAOYA YOSHIDA and HIDEO BABA \\ Department of Gastroenterological Surgery, Graduate School of Medical Sciences, \\ Kumamoto University, Kumamoto, Japan
}

\begin{abstract}
Background: Colorectal cancer is the second most common cause of cancer-related death worldwide. It is well-known that metasta sis to the liver and lung and local recurrences can occur. Additionally, colorectal cancer occasionally metastasizes to other sites. Only a few reports of such metastases have been published and no definitive therapeutic strategies have been proposed for them. Case Report: The case of a 77-year-old man who was diagnosed with rectal cancer is presented. Eighteen months after curative laparoscopic low anterior resection and D3 lymph node resection, an anal fistula metastasis was diagnosed by computed tomography and biopsy. After administering radiotherapy, percutaneous excision of the lesion. was performed. At 21 months from the surgery, the patient is healthy and no recurrence has been found. Conclusion: Metachronous metastasis of a colorectal cancer to an anal fistula is rare. Careful investigation and optimal treatment can result in a disease-free status.
\end{abstract}

Anal fistulas are known to result in abscesses; additionally, primary cancer originates in $0.1 \%$ of anal fistulas $(1,2)$. Metastasis of a cancer to an anal fistula is very rare. There are some reports of synchronous colorectal cancer in an anal fistula; however, metachronous colorectal cancer developing in an anal fistula has rarely been reported (3). We present a case of a patient with rectal cancer who developed a recurrence in an anal fistula and was treated successfully with radiation therapy (RT) and surgery.

This article is freely accessible online.

Correspondence to: Hideo Baba, MD, Ph.D., Department of Gastroenterological Surgery, Graduate School of Medical Sciences, Kumamoto University 1-1-1 Honjo, Kumamoto 860-8556, Japan. Tel: +81 963735212, Fax: +81 963714378, e-mail: hdobaba@kumamoto-u.ac.jp

Key Words: Rectal cancer, anal fistula, recurrence.

\section{Case Report}

A 77-year-old man with a history of having undergone resection of four retroperitoneal liposarcomas in our Department two to nine years previously presented with anaemia (haemoglobin concentration $0.96 \mathrm{~g} / \mathrm{l}$ ) and underwent immediate investigation. Colonoscopy revealed a type 2, advanced, rectal cancer. Contrast-enhanced computer tomography (CECT) showed thickening of the rectum and enlargement of a few regional lymph nodes but no distant metastasis. Additionally, rectal examination revealed an anal fistula on the right side of the anus. Because this fistula was asymptomatic and had a normal gross appearance, we decided to simply follow it up (Figure 1A). Given his history of surgery for liposarcomas in the upper abdomen, we decided to perform laparoscopic low anterior resection with D3 lymph node resection. As expected, there were adhesions in the upper abdomen; however, curative resection was achieved uneventfully. Once surgery-related inflammation had resolved, his drain was removed and he was discharged on postoperative day 23. Pathological examination of the operative specimen revealed moderately differentiated adenocarcinoma that had spread into the muscularis propria. Additionally, two lymph node metastases were detected near the rectum. Eight courses of capecitabine were administered as adjuvant chemotherapy. One year and three months after the surgery, follow-up CECT showed that the previously noted $2-\mathrm{cm}$ mass on the right side of the anus had grown to $3.6 \mathrm{~cm}$ and was now associated with an anal fistula (Figure 1B, C and D). Ring enhancement of the mass suggestive of an abscess was noted. A biopsy confirmed the absence of malignant change.

However, 3 months later, another Magnetic resonance imaging (MRI) revealed that the mass had grown to $5.6 \mathrm{~cm}$ (Figure 2A, B). This time, a biopsy resulted in a diagnosis of adenocarcinoma. This lesion was moderately differentiated adenocarcinoma and had the same pathological characteristics as the primary rectal cancer. It was therefore considered likely to represent a metastasis from that cancer. Because the mass was located very close to the anus, a 

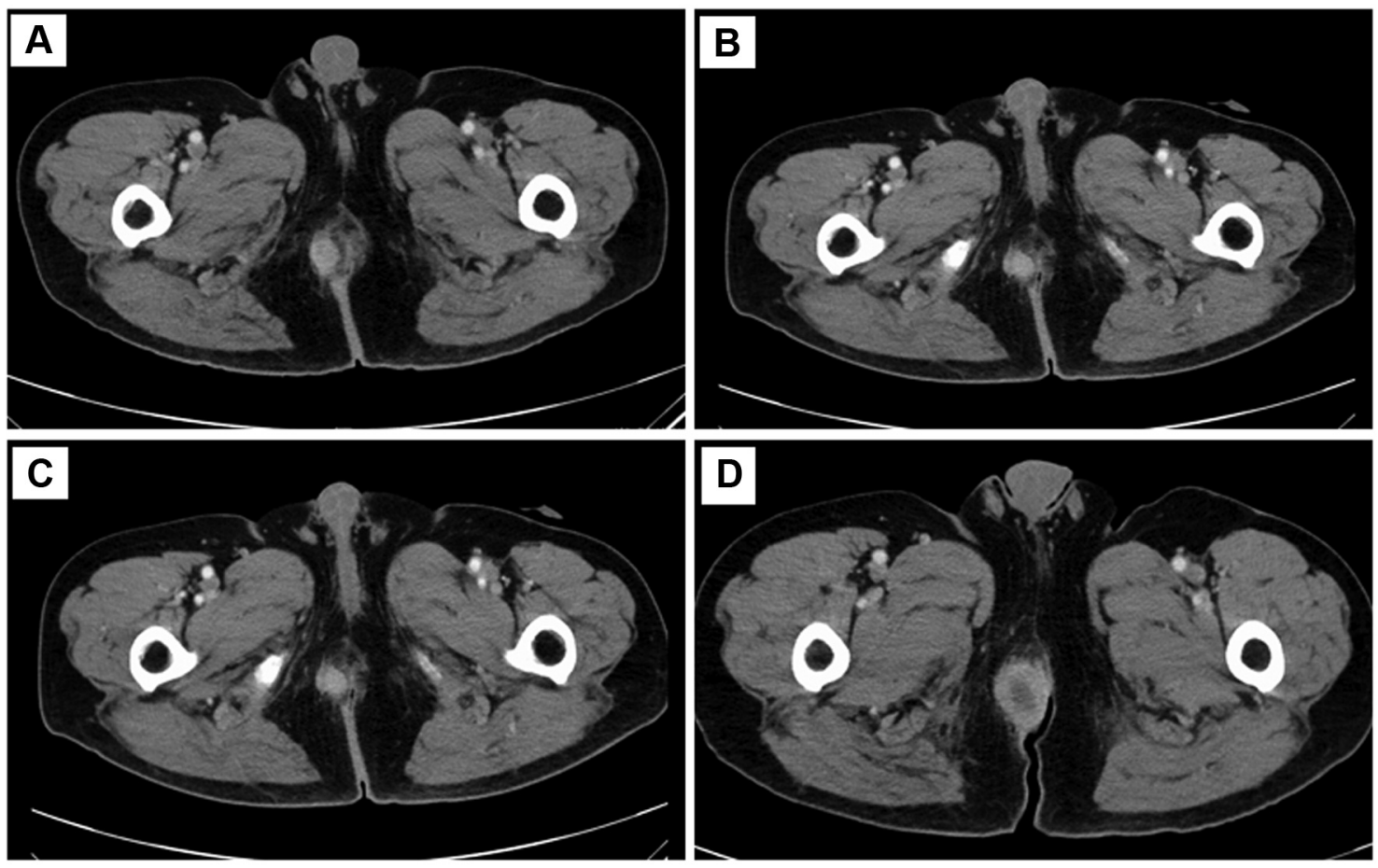

Figure 1. Computed tomography of the development of the anal fistula. A: Before rectal cancer surgery, when we first detected a $2.0 \mathrm{~cm}$ mass in the right side of the anus but it had no signs of fistula. B, C: After surgery, the size of the mass was stable. D: At 1 year and 3 months from surgery, the mass had grown to $3.6 \mathrm{~cm}$, and was also associated with an anal fistula. A biopsy was performed but no malignancy was detected.

decision was made to treat it with RT: The total irradiation dose was $56 \mathrm{~Gy}$. A subsequent CECT showed the mass had shrunk to $4.5 \mathrm{~cm}$, with evidence of necrosis. We then performed percutaneous excision of the lesion with an adequate margin and completely resected the fistula (Figure $3 \mathrm{~A}, \mathrm{~B})$. He was discharged on postoperative day 18 having had no complications. At the time of writing 21 months have elapsed since that surgery, there is no evidence of recurrence, and the patient is healthy.

\section{Discussion}

Carcinoma in an anal fistula is rare, occurring in $0.1-0.3 \%$ of all anal fistulas and accounting for $0.1-0.4 \%$ of colorectal cancer (4). Metastasis to or recurrence of colorectal cancer in an anal fistula is rarer than primary cancer in an anal fistula. Ikeda et al. reported 24 cases of rectal cancer with perianal metastasis, only four of which were metachronous (3-7). In those four cases, the recurrences were found 12-27 months after resection of the primary tumour. We diagnosed a relapse in our patient 15 months after surgery. There was a fistula near the anus when the initial rectal cancer was detected. At that stage, there was no evidence of malignant disease and the fistula was asymptomatic. During postoperative follow-up, the fistula had formed a mass and it enlarged. Gomez et al. reported that recurrences in anal fistulas can only be diagnosed by pathological examination (8). Taking biopsies in all cases of anal fistula and anorectal abscess may be excessively prudent. However, our patient's mass was growing and the fistula was new. Increased or bloody discharge, nodule formation, slow healing, and scar induration may be good indicators for the need for a biopsy (8).

It is believed that colorectal cancer metastasis to an anal fistula is attributable to implantation of cancer cells. To the best of our knowledge, Guiss et al. were the first to report cancer implantation in an anal fistula (in 1954) (9). Cancer implantation in haemorrhoidectomy wounds and in anal injuries caused by using a circular stapler have also been reported $(10,11)$. Our patient had no such injury; however, care must be taken not to create mesorectal or perianal lesions during surgery.

There are no well-established treatments for either synchronous or metachronous metastatic cancer in an anal fistula. In our case, there was no evidence of metastases on the initial presentation, so we performed low anterior resection and D3 lymph node resection. After subsequent adjuvant chemotherapy (capecitabine) for eight courses, we followed up with CECT every 1-3 months. Given that the 

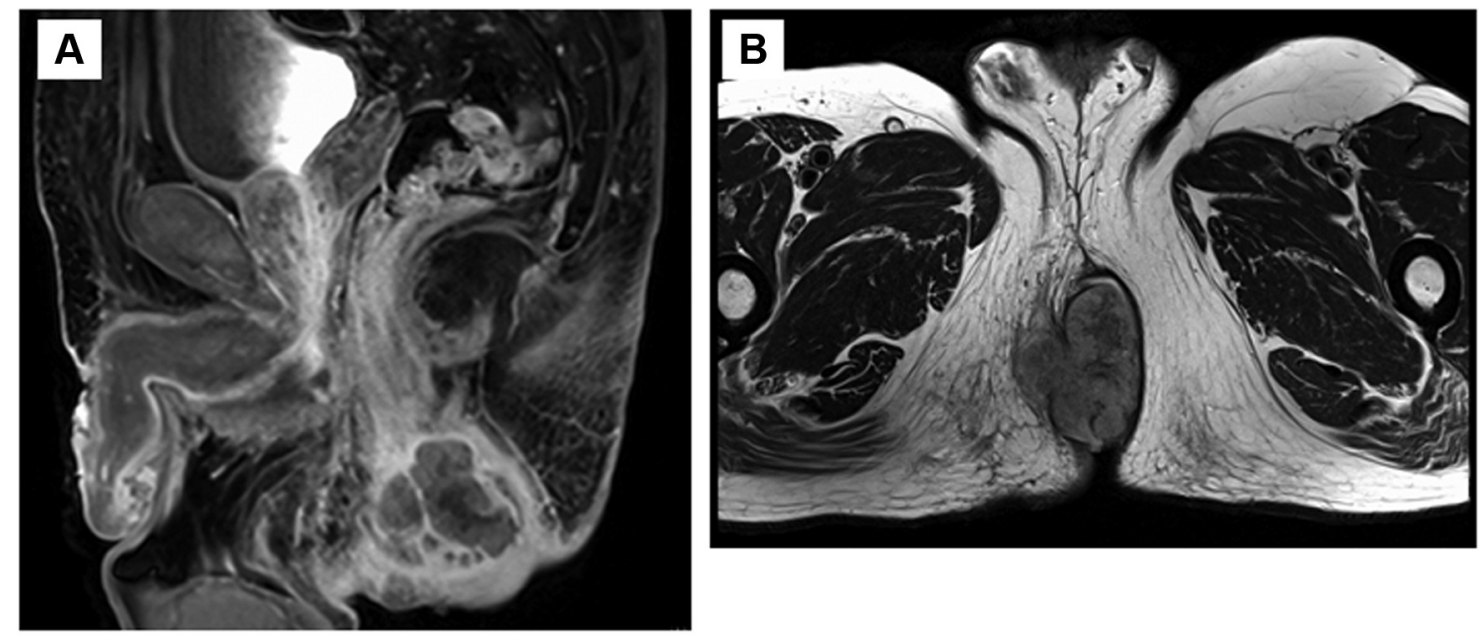

Figure 2. Magnetic resonance imaging at 3 months from the first biopsy. The fistula had grown to $5.6 \mathrm{~cm}$, which was $2.0 \mathrm{~cm}$ larger than 3 months before. The margin was enhanced, and showed a diffusional limitation. A biopsy was again performed, and the anal fistula was diagnosed as moderately to highly differentiated adenocarcinoma, which was an implant from the rectal cancer.
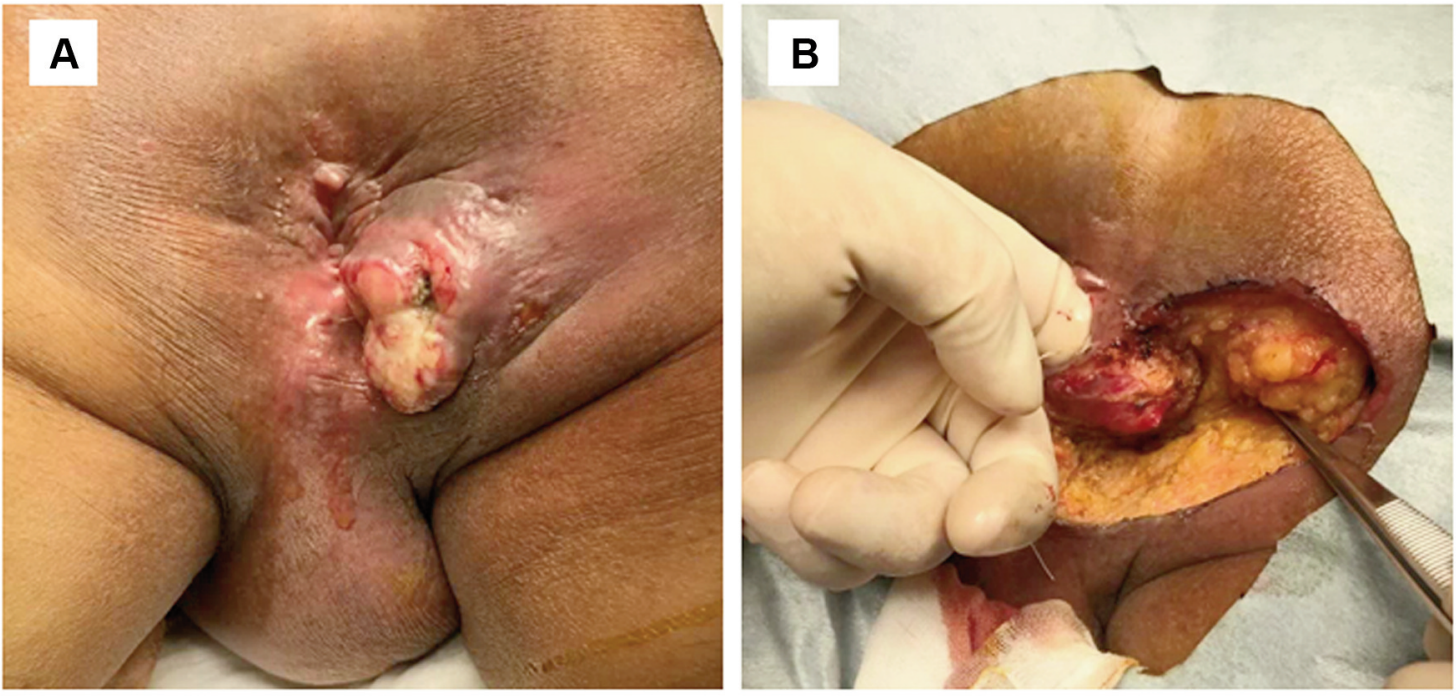

Figure 3. Pre-and perioperative views. A: The patient's anus before the surgery. The mass and the fistula can be seen from the outer surface. B: View after the percutaneous excision. The left forefinger is in the anal canal, which shows there was no damage to it. The mass was exenterated with an adequate margin.

only recurrence we detected was confined to the metastasis in the anal fistula, we considered that surgery was the treatment of choice. We recommended abdominal resection but the patient strongly refused to consent to a colostomy. After 56 Gy of RT, the tumour shrank and some necrosis was apparent. We then performed percutaneous resection of the tumour and anal fistula en bloc. To the best of our knowledge, this sequence of treatment has not been reported previously. ChemoRT has been administered postoperatively or preoperatively in a few cases of synchronous anal fistula and colorectal cancer; none of those patients developed recurrences (3). Furthermore, there are no reports of treatment of such cases with RT alone $(3,12)$. RT may have complications, including radiation proctitis and adhesive 
obstruction; however, obviation of the need for a colostomy provides better quality of life.

\section{Conclusion}

We report a rare case of rectal cancer with a metachronous anal fistula metastasis. Such recurrences are best diagnosed by biopsy. It is important to recognize that implantation into anal regions can occur. Careful investigation and optimal treatment can lead to a disease-free status.

\section{Conflicts of Interest}

There are no conflicts of interest.

\section{Authors' Contributions}

R.K. and Y.M. carried out the experiment. R.K. wrote the manuscript with support from Y.M. Y.M., H.S., K.O., I.M., S.I., Y.B and N.Y. H.B. supervised the project.

\section{Acknowledgements}

The Authors thank Dr Trish Reynolds, MBBS, FRACP, from Edanz Group (https://en-author-services.edanz.com/ac) for editing a draft of this article.

\section{References}

1 Takahashi R, Ichikawa R, Ito S, Mizukoshi K, Ishiyama S, Sgimoto K, Kojima Y, Goto M, Tomiki Y, Yao T and Sakamoto $\mathrm{K}$ : A case of metastatic carcinoma of anal fistula caused by implantation from rectal cancer. Surg Case Rep 1(1): 123, 2015. PMID: 26943447. DOI: 10.1186/s40792-015-0125-2

2 Rollinson PD and Dundas SA: Adenocarcinoma of sigmoid colon seeding into pre-existing fistula in ano. Br J Surg 71(9): 664-665, 1984. PMID: 6478153. DOI: 10.1002/bjs.1800710904

3 Ikeda T, Nanashima A, Ichihara A, Kitamura E, Nagatomo K and Tanaka H: A rare case of rectal cancer with perianal metastasis: a case report. World J Surg Oncol 17(1): 149, 2019. PMID: 31429762. DOI: 10.1186/s12957-019-1692-7
4 Ishiyama S, Inoue S, Kobayashi K, Sano Y, Kushida N, Yamazaki Y and Yanaga K: Implantation of rectal cancer in an anal fistula: report of a case. Surg Today 36(8): 747-749, 2006. PMID: 16865523. DOI: 10.1007/s00595-006-3236-3

5 Isbister WH: Unusual 'recurrence' sites for colorectal cancer. Dig Surg 17(1): 81-83, 2000. PMID: 10720837. DOI: 10.1159/00001 8805

6 Gravante G, Delogu D and Venditti D: Colosigmoid adenocarcinoma anastomotic recurrence seeding into a transsphincteric fistula-in-ano: a clinical report and literature review. Surg Laparosc Endosc Percutan Tech 18(4): 407-408, 2008. PMID: 18716545. DOI: 10.1097/SLE.0b013e3181693346

7 Wakatsuki K, Oeda Y, Isono T, Yoshioka S, Nukui Y, Yamazaki $\mathrm{K}$, Nabeshima $\mathrm{S}$ and Miyazaki M: Adenocarcinoma of the rectosigmoid colon seeding into pre-existing anal fistula. Hepatogastroenterology 55(84): 952-955, 2008. PMID: 18705305.

8 Gomes RM, Kumar RK, Desouza A and Saklani A: Implantation metastasis from adenocarcinoma of the sigmoid colon into a perianal fistula: a case report. Ann Gastroenterol 27(3): 276-279, 2014. PMID: 24975988

9 Guiss RL: The implantation of cancer cells within a fistula in ano: case report. Surgery 36(1): 136-139, 1954. PMID: 13178955.

$10 \mathrm{Hsu}$ TC and Lu IL: Implantation of adenocarcinoma on hemorrhoidectomy wound. Int J Colorectal Dis 22(11): 14071408, 2007. PMID: 16804669. DOI: 10.1007/s00384-006-0179-5

11 Norgren J and Svensson JO: Anal implantation metastasis from carcinoma of the sigmoid colon and rectum - a risk when performing anterior resection with the EEA stapler? Br J Surg 72(8): 602, 1985. PMID: 4027530. DOI: 10.1002/bjs.1800720806

12 Sasaki S, Sugiyama M, Nakaji Y, Nakanishi R, Nakashima Y, Saeki H, Oki E, Oda Y and Maehara Y: Anal metastasis of rectal cancer-adenocarcinoma of squamous cells: a case report and literature review. Surg Case Rep 3(1): 55, 2017. PMID: 28432678. DOI: $10.1186 / \mathrm{s} 40792-017-0319-\mathrm{x}$ 